\title{
Customização intraoperatória de endoprótese para correção de fístula aortoentérica em contexto de urgência: um relato de caso
}

\author{
Intraoperative endoprosthesis customization for repair of an aortoenteric fistula in an \\ emergency context: a case report
}

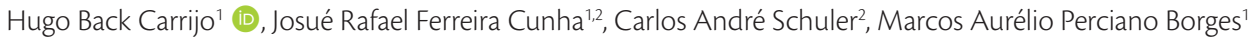

\begin{abstract}
Resumo
A fístula aortoentérica é uma grave condição clínica, e seu manejo permanece sendo um grande desafio técnico aos cirurgiões. A abordagem por cirurgia convencional nesses casos está relacionada a altos índices de morbimortalidade. A cirurgia endovascular apresenta-se como uma ótima alternativa nesses casos; contudo, por não se tratar de aorta nativa, a anatomia pode não ser compatível com os dispositivos endovasculares comercialmente disponíveis, fazendo-se necessário, em casos de urgência, a utilização de dispositivos modificados pelo cirurgião. O caso relatado reporta uma fístula aortoentérica secundária, tratada em situação de urgência por técnica endovascular com dispositivo modificado.
\end{abstract}

Palavras-chave: fístula aortoentérica; aneurisma de aorta; procedimentos endovasculares; endopróteses modificadas.

\begin{abstract}
Aortoenteric fistula is a severe clinical condition and its management remains a major technical challenge for surgeons. In these cases, the conventional surgical approach is associated with high rates of morbidity and mortality. Endovascular surgery is an excellent option in these cases, but considering that the aorta has been treated previously, anatomy may not be compatible with commercially available endovascular devices and so physician-modified endografts may be needed in urgent cases. The case reported involves a secondary aortoenteric fistula, treated on an emergency basis with endovascular techniques, using a physician-modified endograft.
\end{abstract}

Keywords: aortoenteric fistula; aortic aneurysm; endovascular procedures; modified endografts.

Como citar: Carrijo HB, Cunha JRF, Schuler CA, Borges MAP. Customização intraoperatória de endoprótese para correção de fístula aortoentérica em contexto de urgência: um relato de caso. J Vasc Bras. 2021;20:e20200179. https:// doi.org/10.1590/1677-5449.200179

${ }^{1}$ Hospital de Base do Distrito Federal - HBDF, Brasília, DF, Brasil.

Instituto de Cardiologia do Distrito Federal - ICDF, Brasília, DF, Brasil.

Fonte de financiamento: Nenhuma.

Conflito de interesse: Os autores declararam não haver conflitos de interesse que precisam ser informados.

Submetido em: Outubro 21, 2020. Aceito em: Dezembro 07, 2020.

O estudo foi realizado no Instituto de Cardiologia do Distrito Federal (ICDF), Brasília, DF, Brasil. 


\section{INTRODUÇÃO}

A fístula aortoentérica é uma complicação rara, mas que consiste em uma grave ameaça à vida. Em que pese ao seu longevo primeiro relato, por Ashley Cooper, datar de $1818^{1}$, ainda hoje representa um grande desafio terapêutico.

São classificadas como primárias e secundárias, sendo as primárias bastante incomuns, advindas de aneurismas volumosos, que causam a erosão do trato entérico adjacente, e as secundárias mais comuns, usualmente oriundas de enxertos aórticos com prótese, com prevalência entre 0,3 a $1,6 \%$ dos casos $^{2}$. O quadro clínico inclui dor abdominal, hemorragia gastrointestinal e presença de massa abdominal pulsátil. Contudo, o diagnóstico costuma ser desafiador, visto que essa tríade clássica está presente em apenas $23 \%$ dos pacientes ${ }^{3,4}$.

O diagnóstico normalmente é feito por angiotomografia, método que apresenta ampla vantagem quando comparado a outros exames de imagem, visto ser exame de alta resolução, fácil acesso, pouco invasivo e com baixo tempo de aquisição. A cirurgia convencional para a correção da fístula aortoentérica secundária é tradicionalmente baseada na confecção de bypass extra-anatômico, ligadura da aorta e remoção do enxerto previamente interposto. No entanto, essa abordagem está associada a alta morbimortalidade: 25 a $90 \%$ de mortalidade operatória e 5 a $25 \%$ de amputação de membros inferiores ${ }^{5,6}$. Nesse contexto, a abordagem endovascular surge como uma opção terapêutica de grande valia para a correção de fístula aortoentérica, visto a gravidade desses pacientes e a morbidade do procedimento via cirurgia convencional.

No entanto, há de se levar em conta a anatomia complexa que muitos desses pacientes apresentam, em que as endopróteses disponíveis no momento da abordagem não se adequam aos critérios anatômicos do paciente. Dessa forma, modificações nas endopróteses são realizadas e descritas, com sucesso, por alguns autores, seja com ressecção de um segmento ou confecção de fenestrações. Essa abordagem é habitualmente reservada aos casos de urgência, para viabilizar a correção endovascular ${ }^{7-9}$.

O protocolo foi aprovado pelo Comitê de Ética de nossa instituição (parecer número 4.748.984).

\section{RELATO DE CASO}

Um paciente do sexo masculino, de 66 anos, iniciou quadro de hemorragia digestiva alta, com dois episódios de hematêmese há dois dias da admissão hospitalar, mantendo vários episódios de melena desde então. Submetido, há 15 anos, à correção aberta por derivação aorto-femoral direito e ilíaca externa esquerda com prótese de Dacron, apresentava também como antecedentes hipertensão arterial sistêmica e doença pulmonar obstrutiva crônica.

Foi realizada angiotomografia de abdome que visualizou dilatação sacular na aorta abdominal infrarrenal em região proximal do bypass, fazendo interface com a quarta porção do duodeno, medindo $51 \times 41 \mathrm{~mm}$, sugestivo de pseudoaneurisma de anastomose proximal do enxerto, associado à fístula aortoentérica (Figuras 1 e 2).

Por se tratar de fístula aortoentérica secundária, associada à prévia abordagem abdominal em paciente em grave estado clínico, optou-se por abordagem endovascular da lesão. Contudo, ao se realizar a reconstrução da angiotomografia e tomadas as medidas para planejamento do reparo endovascular, constatouse que os parâmetros anatômicos eram inadequados, por dois motivos principais:

1) Diâmetro do colo proximal de $36 \mathrm{~mm}$, impossibilitando a utilização de endopróteses bifurcadas. Vale lembrar que os maiores diâmetros disponíveis são da ordem de $36 \mathrm{~mm}$;

2) Distância entre a artéria renal mais baixa e bifurcação da prótese de Dácron, utilizada no

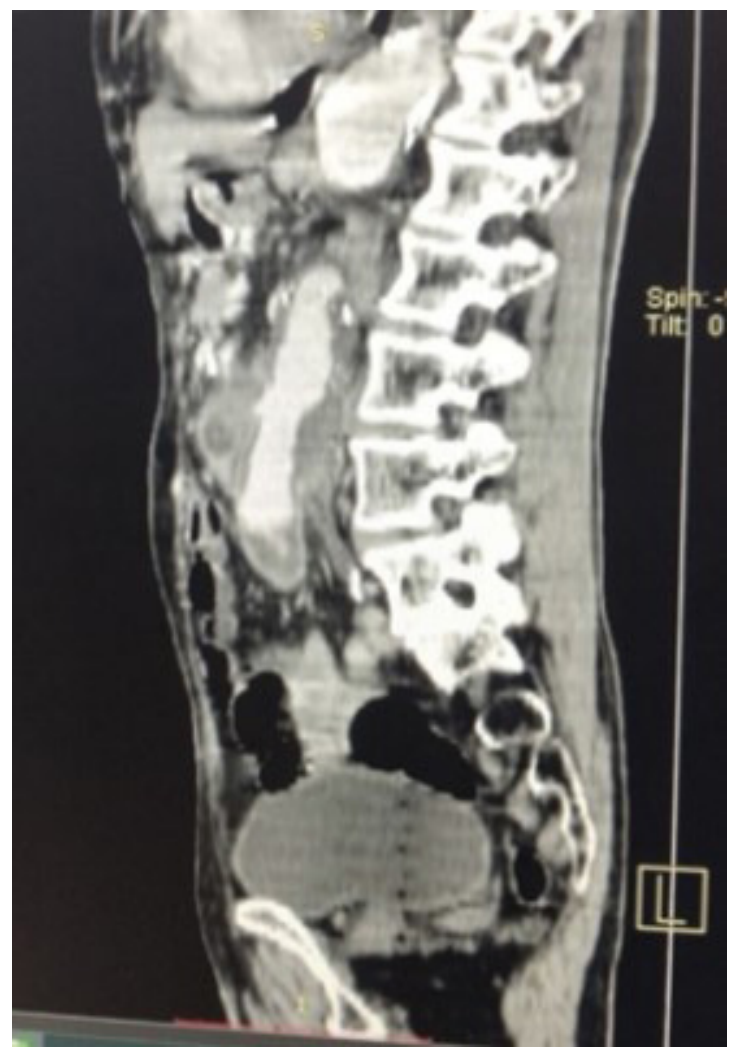

Figura 1. Angiotomografia de abdome em corte sagital demonstrando fistula aortoentérica em região da anastomose proximal de bypass aórtico prévio. 
bypass aorto-femoral direito e ilíaca externa esquerda, de $89 \mathrm{~mm}$, impossibilitando o uso de endoprótese de aorta torácica, visto que os dispositivos mais curtos apresentam $100 \mathrm{~mm}$ de extensão.

Sob essas condições anatômicas, portanto, não há dispositivo endovascular comercialmente disponível para empregar prontamente.

Dada a urgência e gravidade do caso, além da impossibilidade de customização de dispositivo endovascular, optou-se pela modificação intraoperatória da endoprótese torácica, Valiant Captivia Medtronic 40x167 mm (Medtronic, Minnesota, EUA).

Em primeiro lugar, foi realizada a liberação do dispositivo em mesa cirúrgica auxiliar "backtable" (Figura 3). Posteriormente, foi liberado o primeiro estágio da endoprótese Valiant Captivia Medtronic, mantendo fixo o seu anel aberto proximal. Após isso, foram realizadas as medidas do dispositivo e ressecção da extensão distal excedente com lâmina de bisturi (Figura 4).

Em seguida, foi realizado o rencapamento da endoprótese, com auxílio de fita cardíaca, de forma lenta e progressiva, até a total cobertura de toda a extensão do dispositivo pelo sistema de entrega e liberação (Figura 5). Para o procedimento, foi realizada dissecção da artéria femoral direita com passagem de introdutor $7 \mathrm{~F}$ e passagem de introdutor $5 \mathrm{~F}$ em femoral esquerda por punção ecoguiada. A endoprótese foi introduzida pela femoral comum direita sob fioguia Lunderquist (Cook Group Inc., Indiana, EUA), posicionada em situação infrarrenal, recobrindo o pseudoaneurisma de anastomose proximal do bypass aórtico prévio. Não houve intercorrência para a liberação da endoprótese e o controle final angiográfico

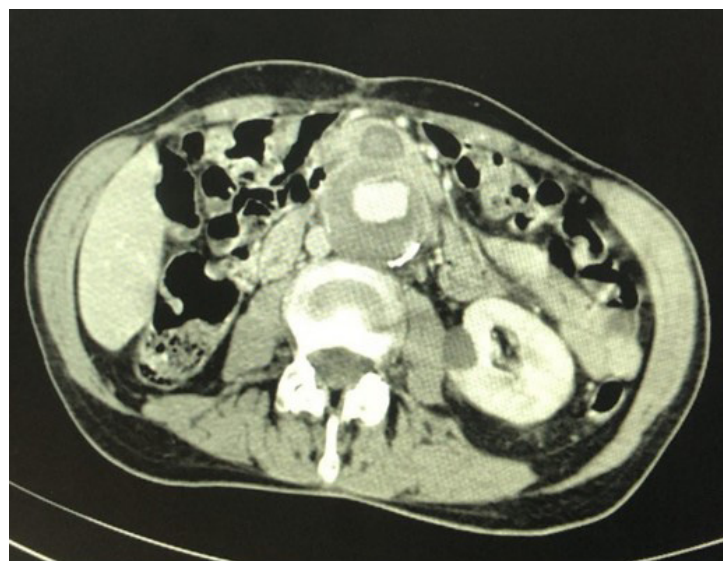

Figura 2. Angiotomografia de abdome em corte axial demonstrando fístula aortoentérica em região proximal da anastomose proximal de bypass aórtico prévio. demonstrou exclusão completa do pseudoaneurisma e resolução da fístula aortoentérica (Figura 6).

O paciente apresentou boa evolução no pós-operatório, recebendo alta hospitalar após $72 \mathrm{~h}$ de internação, com boa aceitação da dieta oral, sem intercorrências e sem novos episódios de sangramentos.

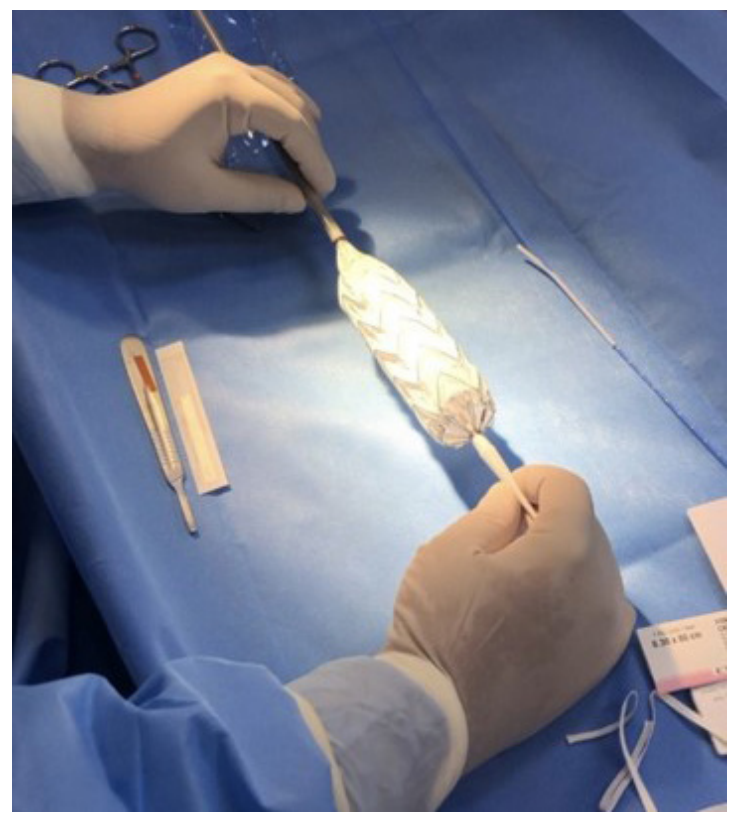

Figura 3. Liberação da endoprótese Valiant Captivia Medtronic (Medtronic, Minnesota, EUA).

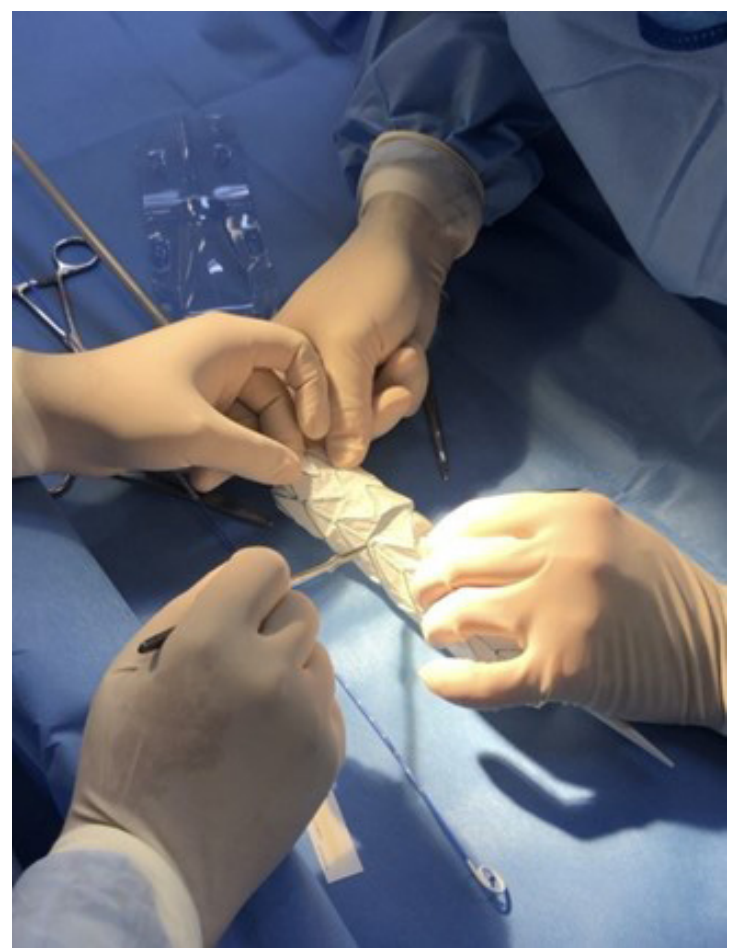

Figura 4. Ressecção de segmento distal da endoprótese Valiant Captivia Medtronic (Medtronic, Minnesota, EUA). 


\section{DISCUSSÃO}

As fístulas aortoentéricas são uma das complicações mais graves do aneurisma de aorta e costumam apresentar altas taxas mortalidade pós-operatória ${ }^{10}$.

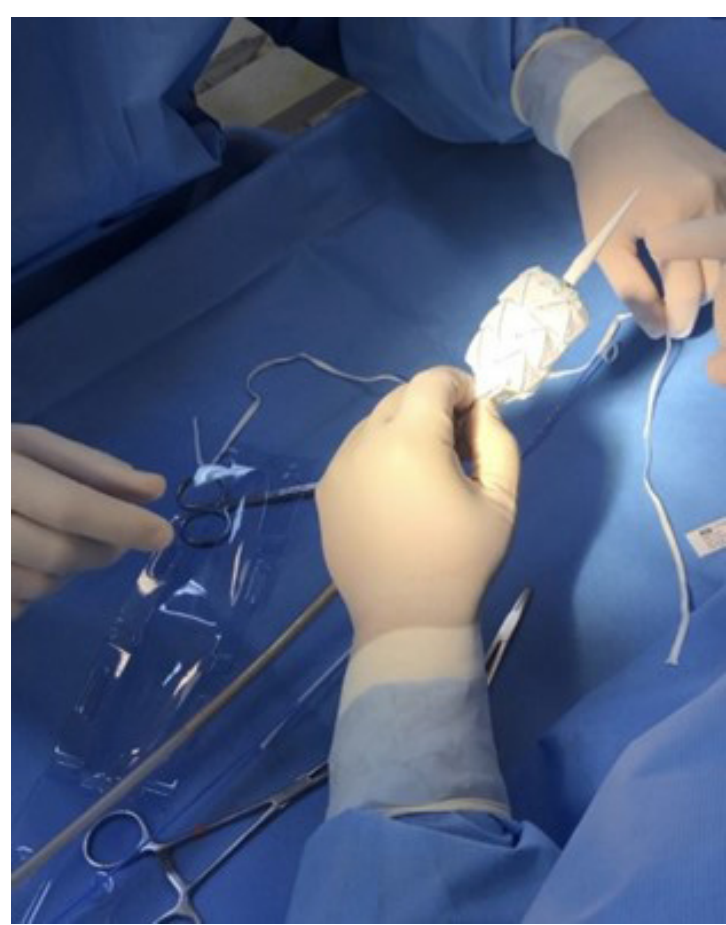

Figura 5. Rencapamento da endoprótese Valiant Captivia Medtronic (Medtronic, Minnesota, EUA).

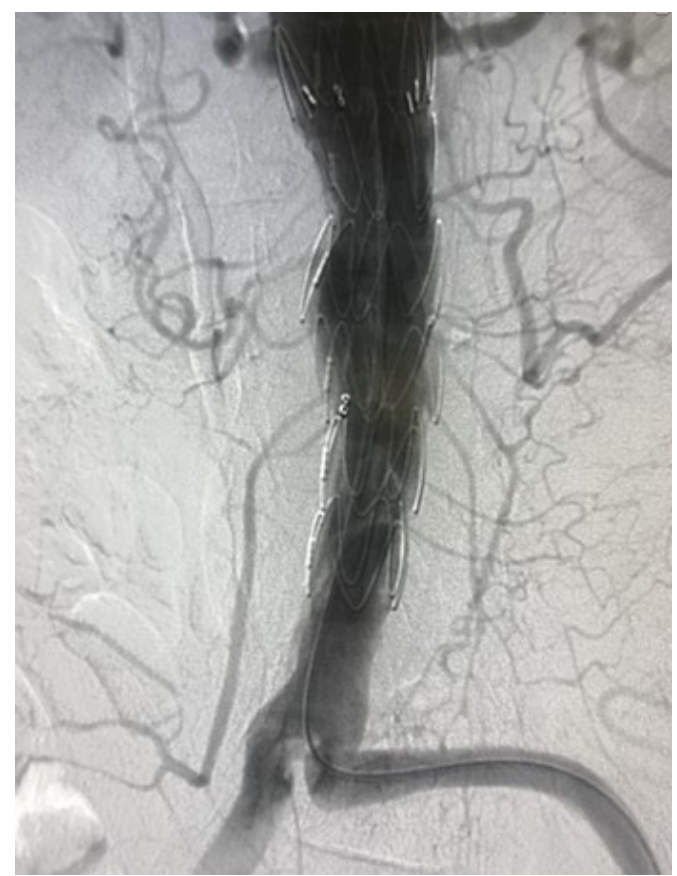

Figura 6. Angiografia final demonstrando exclusão completa do pseudoaneurisma e resolução da fístula aortoentérica.
A correção aberta está associada a grande morbidade cirúrgica, tendo em vista a dificuldade técnica de abordagem em abdome previamente operado e o risco de sangramento e contaminação significativo, associado à baixa patência da revascularização extra-anatômica ${ }^{11}$.

Uma revisão que analisou 216 artigos com um total de 823 pacientes com fistula aortoentérica demonstrou que a mortalidade intra-hospitalar na abordagem endovascular é menor que na abordagem aberta $(7,1 \%$ contra $33,9 \%$, respectivamente), sem diferenças estatísticas nas taxas de recorrência de fistula aortoentérica entre as abordagens. $\mathrm{O}$ benefício de sobrevida entre os tipos de abordagem diminuiu progressivamente ao longo dos anos, secundário a maior taxa de infecção na abordagem endovascular, porém mantendo, ainda assim, maior sobrevida nesse grupo ${ }^{12}$.

A correção endovascular é uma opção para tratamento das fistulas aortoentéricas, especialmente em pacientes com comorbidades e quadro clínico grave. Como o contexto em que é realizado é de urgência, há artifícios que podem ser utilizados para adequação do material disponível no momento para a correção endovascular. Há diversos estudos relatando casos de modificação intraoperatória de endopróteses aórticas, seja com ressecção de um segmento ou mesmo confecção de fenestras ${ }^{13,14}$.

A realização dessas modificações no momento da correção ganha relevância especialmente em casos de anatomia complexa, haja vista que dispositivos personalizados requerem semanas para serem produzidos $^{14}$. Levando-se em conta os critérios anatômicos "on label" dos dispositivos para correção dos aneurismas de aorta, alguns autores discorrem que menos da metade dos pacientes com aneurismas pararrenais e toracoabdominais preenchem os critérios para correção com as endopróteses "off the shelf", fazendo com que a personalização dos dispositivos tenha seu papel em casos selecionados ${ }^{14,15}$.

Sweet et al. ${ }^{16}$ demonstraram que as próteses modificadas pelo cirurgião para tratamento de aneurismas toracoabdominais apresentaram, em curto prazo, resultados comparáveis a dispositivos manufaturados, podendo ser utilizadas de forma segura e eficaz em pacientes com condições clínicas desfavoráveis para o procedimento cirúrgico convencional.

A maior preocupação em relação à customização desses dispositivos é quanto à durabilidade, visto a carência de volume de estudos que certifiquem a durabilidade e a eficácia desses dispositivos modificados a longo prazo. Essa preocupação também estava presente no início da terapia endovascular dos aneurismas de aorta. Contudo, apesar de não se contar 
com evidência em larga escala, algumas publicações ratificam a segurança dos dispositivos modificados ao longo do tempo.

Starnes et al. ${ }^{17}$ realizaram o seguimento por 12 meses de 59 pacientes submetidos à correção de aneurismas justarrenais de aorta, com dispositivos modificados por cirurgiões. Nesse período, foram identificados apenas dois endoleaks tipo III, um endoleak tipo IB e nenhum endoleak tipo IA.

Em recente publicação, os mesmos autores relataram o seguimento de 7 anos de correção de aneurisma justarrenal. No mesmo sentido, o relato observa endopróteses modificadas por cirurgião. Durante 5 anos, os sucessivos controles tomográficos não demostraram falhas estruturais, migração ou endoleaks, constatando-se, pelo contrário, um remodelamento aórtico com redução de diâmetro do saco aneurismático. No sétimo ano de seguimento, o paciente veio a falecer de causas não relacionadas a doença da aorta. $\mathrm{O}$ estudo post-mortem demonstrou a fratura de apenas uma haste de stent de endoprótese, sem causar migração do dispositivo, ratificando a efetividade e segurança a longo prazo de dispositivos modificados por cirurgiões ${ }^{18}$.

Não há dúvidas de que as endopróteses customizadas, ou "off the shelf", devam ser priorizadas, mas a expertise em modificar os dispositivos deve estar presente no arsenal terapêutico dos cirurgiões, principalmente nas urgências e em casos em que os dispositivos não atendem as exigências anatômicas do paciente. Também não se pode esquecer das peculiaridades da medicina brasileira, com um cenário no qual nem todos os pacientes têm acesso irrestrito ao aparato endovascular, e, mais uma vez, nessas situações, a habilidade em modificar os dispositivos endovasculares pode representar uma significativa diferença no prognóstico dos pacientes.

\section{REFERÊNCIAS}

1. Cooper A. The lectures on the principles and practice of surgery with additional notes and cases by Frederick Tyrrell. London: Thomas \& George Underwood; 1824. (vol. 2).

2. PeckJJ, Eidemiller LR. Aortoenteric fistulas. Arch Surg. 1992;127(10):1191 3. http://dx.doi.org/10.1001/archsurg.1992.01420100049008. PMid:1417484.

3. Bergqvist $D$. Arterioenteric fistula: review of a vascular emergency Acta Chir Scand. 1987;153(2):81-6. PMid:3303777.

4. Bernhard VM. Aortoenteric fistula. Orlando, FL: Grune\&Stratton; 1985. p. 513-25.
5. Umpleby HC, Britton DC, Turnbull AR. Secondary aortoenteric fistulae: a surgical challenge. Br J Surg. 1987;74(4):256-9. http:// dx.doi.org/10.1002/bjs.1800740411. PMid:3580796.

6. Kinney EV, Kaebnick HW, Mitchell RA, Jung MT. Repair of mycotic paravisceral aneurysm with a fenestrated stent graft. J Endovasc Ther. 2000;7(3):192-7. http://dx.doi.org/10.1177/152660280000700304. PMid:10883955.

7. Chenu C, Marcheix B, Barcelo C, Rousseau H. Aorto-enteric fistula after endovascular abdominal aortic aneurysm repair: case report and review. Eur J Vasc Endovasc Surg. 2009;37(4):401-6. http:// dx.doi.org/10.1016/j.ejvs.2008.11.037. PMid:19211278.

8. Fernandez Prendes C, Del Castro Madrazo JA, Padron Encalada CE, Dominguez MR, Camblor Santervas LA, Perez MA. Hybrid repair of an innominate artery mycotic aneurysm with an "on-the-table" customized endograft. Ann Vasc Surg. 2019;59:311.e5-9. http:// dx.doi.org/10.1016/j.avsg.2018.12.097. PMid:30802585.

9. Cochennec $\mathrm{F}$, Kobeiter $\mathrm{H}$, Gohel $\mathrm{M}$, et al. Early results of physician modified fenestrated stent grafts for the treatment of thoracoabdominal aortic aneurysms. Eur J Vasc Endovasc Surg. 2015;50(5):58392. http://dx.doi.org/10.1016/j.ejvs.2015.07.002. PMid:26259766.

10. Chung J. Management of aortoenteric fistula. Adv Surg. 2018;52(1):15577. http://dx.doi.org/10.1016/j.yasu.2018.03.007. PMid:30098611.

11. Mathias J, Mathias E, Jausset F, et al. Aorto-enteric fistulas: a physiopathological approach and computed tomography diagnosis. Diagn Interv Imaging. 2012;93(11):840-51. http://dx.doi. org/10.1016/j.diii.2012.07.003. PMid:23092721.

12. Kakkos SK, Bicknell CD, Tsolakis IA, Bergqvist D. Editor's choice - management of secondary aorto-enteric and other abdominal arterio-enteric fistulas: a review and pooled data analysis. Eur J Vasc Endovasc Surg. 2016;52(6):770-86. http://dx.doi.org/10.1016/j. ejvs.2016.09.014. PMid:27838156.

13. Voorhoeve R, Moll FL, Bast TJ. The primary aortoentericfistula in the Netherlands - the unpublished cases. Eur J Vasc Endovasc Surg. 1996;11(4):429-31. http://dx.doi.org/10.1016/S1078-5884(96)801766. PMid:8846177.

14. Antoniou GA, Koutsias S, Antoniou SA, Georgiakakis A, Lazarides $M K$, Giannoukas AD. Outcome after endovascular stent graft repair of aortoenteric fistula: a systematic review. J Vasc Surg. 2009;49(3):7829. http://dx.doi.org/10.1016/j.jvs.2008.08.068. PMid:19028054.

15. Oderich GS. Novos horizontes na doença aórtica: os últimos legado de um inovador visionário. J Endovasc Ther. 2015;22(1):139-45. http://dx.doi.org/10.1177/1526602814566180. PMid:25775695.

16. Sweet MP, Starnes BW, Tatum B. Endovascular treatment of thoracoabdominal aortic aneurysm using physician-modified endografts. J Vasc Surg. 2015;62(5):1160-7. http://dx.doi.org/10.1016/j. jvs.2015.05.036. PMid:26194816

17. Starnes BW, Heneghan RE, Tatum B. Midterm results from a physician-sponsored investigational device exemption clinical trial evaluating physician-modified endovascular grafts for the treatment of juxtarenal aortic aneurysms. J Vasc Surg. 2017;65(2):294-302. http://dx.doi.org/10.1016/j.jvs.2016.07.123. PMid:27687323.

18. Hurd JR, Tatum B, Grillo J, et al. Long-term durability of a physicianmodified endavascular graft. J Vasc Surg. 2020;71(2):628-34. http:// dx.doi.org/10.1016/j.jvs.2019.04.471. PMid:31401117. 
Correspondência

Hugo Back Carrijo

Instituto Hospital de Base do Distrito Federal - IHB, Unidade de Cirurgia Vascular - SMHS Área especial quadra 1

CEP 70658-700- Brasília (DF), Brasil Tel.: (61) 99643-8803 E-mail: hbcarrijo@gmail.com

Informações sobre os autores

HBC - Residente de Cirurgia Vascular, Hospital de Base do Distrito Federal (HBDF); Cirurgião geral, Hospital das Clínicas, Faculdade de Medicina, Universidade de São Paulo (HC-FMUSP).

JRFC - Cirurgião vascular e endovascular, Hospital de Base do Distrito Federal (HBDF); Cirurgião geral, Hospital Universitário de Brasília (HUB); Médico assistente, HBDF, Instituto de Cardiologia do Distrito Federal (ICDF). CAS - Cirurgião geral, vascular e endovascular, Hospital Regional de São JoséSC; Médico assistente, Instituto de Cardiologia do Distrito Federal (ICDF). MAPB - Cirurgião vascular formado, Hospital de Base do Distrito Federal (HBDF); Médico assistente, HBDF.

Contribuições dos autores Concepção e desenho do estudo: HBC, CAS Análise e interpretação dos dados: HBC, CAS, JRFC, MAPB

Coleta de dados: HBC Redação do artigo: $\mathrm{HBC}, \mathrm{CAS}$ Revisão crítica do texto: $\mathrm{HBC}, \mathrm{CAS}, \mathrm{JRFC}, \mathrm{MAPB}$ Aprovação final do artigo*: HBC, CAS, JRFC, MAPB Análise estatística: Não se aplica. Responsabilidade geral pelo estudo: HBC, CAS

*Todos os autores leram e aprovaram a versão final submetida ao | Vasc Bras. 